\title{
The surface modification of a polyethylene (PE) film using an air Coplanar barrier discharge plasma at atmosphere pressure
}

\author{
Kun WANG ${ }^{1,2, a}$, Jian $\mathrm{LI}^{1,2, b}$, Shi-qing WANG ${ }^{1,2, c}$ \\ ${ }^{1}$ Southwestern Institute of Physics, Chengdu, 610041, China \\ ${ }^{2}$ Engineering and Technical College of Chengdu University of Technology, Leshan, 614007, China \\ a wangkun6236@sina.com, ${ }^{b}$ lijwk@126.com, c wsq6222@126.com
}

Keywords: Air coplanar barrier discharge, Polyethylene, Surface modification, Wettability

\begin{abstract}
In this paper, modification of the surface properties of polyethylene (PE) films is studied using air coplanar barrier discharge plasma at atmosphere pressure. The diffuse plasma appears on the surface of the device. The treated samples are examined by water contact angle measurements, fourier transform infrared attenuated total reflection spectroscopy (FTIR-ATR), X-ray photoelectron spectroscopy (XPS) and scanning electron microscopy(SEM). With treatment time increasing, the water contact angle changes from the value of $92^{\circ}$ of the untreated PE surface to the minimum value of $51^{\circ}$ after treatment time of $50 \mathrm{~s}$. The results show some oxidized species are introduced into the samples surface by the plasma treatment and the changeable tendency of the water contact angle with the treatment time is the same as that of the $\mathrm{O}$ concentration on the treated sample surface. From the SEM, the surface roughness of PE samples increases with the treatment time increasing.
\end{abstract}

\section{Introduction}

Dielectric barrier discharges (DBDs) occur in configureurations which are characterized by a dielectric barrier layer between conducting electrodes. There are three basic configureurations for generating DBDs[1]. The first is the volume discharge (VBD), which is consisted of two parallel plates with the gas gap distance is about several millmeters. A VBD device consists of two parallel dielectric plates with electrodes outside fitted. As is shown in Figureure 1, the VBD is characterized by initiation in a uniform field with fixed gas gap, while SBD propagates in a non-uniform field from the surface electrode along the dielectric surface with gas gap unfixed.The second is the surface discharge (SBD), a plane dielectric with an electrode on one surface and a metallic cover on its reverse side. The third is coplanar barrier discharge (CBD), which not only has the advantages of DBD, but also has the advantages of itself, such as the lower spark ignition voltage, easily formation of the discharge, etc. In all the basic configureurations the DBDs consist of discharge pulses with a duration of about $10 \mathrm{~ns}[2]$. The volume DBDs are used on a large industrial scale to increase the properties of polymers, films and other materials. It have been tested for atmospheric pressure plasma deposition and have found a number of industrial applications, such as the industrial ozone generation, ultraviolet (UV) or vacuum ultraviolet (VUV) excimer radiation in excimer lamps. But it has some deficiencies for treatment of large area substrates, which can be easily eliminated by using the coplanar barrier discharge structure. Both surface barrier discharge (SBD) and coplanar barrier discharge (DBD) are promising for surface engineering at atmospheric pressure. They are already been used for surface modification[1], plasma cleaning[2] and film deposition[3].

Polymers, which play an increasing role as structural materials, are applied more and more widely in industry in recent years. A lot of polymer properties are well known, such as low surface energy, thermal stability, low friction and hydrophobic. These properties are maybe anticipated in a few applications, while they are defects for other applications and have to be overcome. Recently, modification of the surface properties of polymers becomes a subject, which plenty of researchers are interested in. The usual two means including physical and chemical methods are adopted to modify the surface properties of polymers. However, the chemical method is water-wasting and easily causes 
environment pollution. In contrast, the physical method is paid more and more attention in recent years.

Plasma technique used to modify polymer surfaces is of great and increasing industrial practicability [4] because it can uniformly modify the surface of the treated samples without changing the matrix properties. For example, Chaozong Liu et al [5] had reported that, the AFM examination of pre- and post-plasma treated polymer surfaces revealed that only the outermost layer was affected by dielectric barrier discharge (DBD) plasma treatment. S. Guruvenket et al. [6] had modified polystyrene and PE using microwave electron cyclotron resonance plasma to improve the wettability of the two kinds of polymer. Coen et al. [7] had modified polypropylene (PP), polymethylmethacrylate, polytetrafluoroethylene (PTFE) and polyethyletetraphalate (PET) under high vacuum conditions. The results suggested that plasma treatment could cause topographical modification on the treated polymer surface. S. Bhowmik et al. [8] had studied the wettability and physicochemical characteristics when PP film was exposed to a DC glow discharge on the condition of airflow by different electrode structure.

CBD is proved to be a promising technology in the modification of surface properties of polymers in recent years. The mean electron energy in CBD plasma is in the range of 0-10 eV [9], while the chemical binding energy of polymers is less than or equals to $10 \mathrm{eV} \mathrm{[10].} \mathrm{Therefore,}$ energetic particles in CBD can break the chemical bonds of polymers. In addition, to modify the surface properties of polymers without expensive vacuum system is another advantage of CBD. Nowadays, CBD has been used widely to modify the surface of polyimide, PP, PTFE, poly (methylpentene), and so on $[11,12]$.

The aim of this research was to create a diffuse layer of coplanar barrier discharge plasma at atmospheric pressure in air. It is believed that for many surface treatment applications, such a thin surface layer of plasma may be more useful than the other configureurations. We explored a coplanar barrier discharge apparatus.The parameters of the apparatus are optimized and proposed experimentally. The lamps with CBD structure is designed and cmpared with those with DBD sturucture. The polyethylene (PE) films are treated with the CBD device.

\section{Experimental setup}

Figure 1 shows the schematic diagram of the experiment setup. Coplanar barrrier discharge plasma appears on the surface of the device. The electrode and the gap distance is 0.5 and $1 \mathrm{~mm}$. Figure 2 shows the picture of the neon and argon coplanar barrier discharge.

The contact angle measurement system used is OCAH200. The data are obtained with 5 point average of the water contact angle measurements obtained from the same sample of the area of 60 $\mathrm{mm} \times 60 \mathrm{~mm}$. The FTIR system used is AVATAR 360FT-IR, the accessory used is ATR with the wave number range of 400-4000 cm-1, which examines the evidence for the induction of new functional groups in the surface of PE films. The XPS system used is Kratos Analytical Amicus, which analyzes the concentration of the new elements introduced into the surface of PE films. The SEM system used is JSM-5600LV, which explores the surface morphology of PE samples.
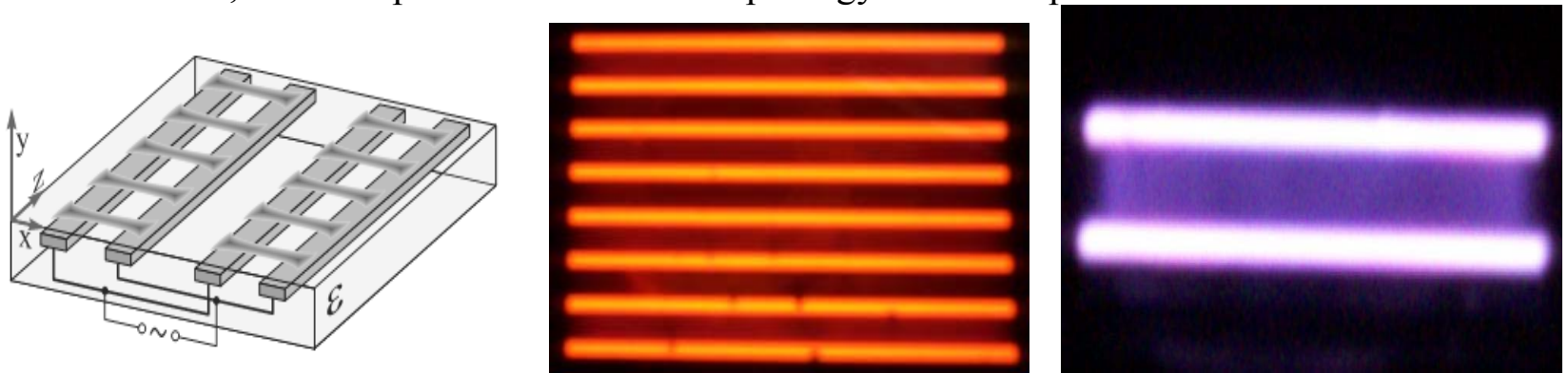

Fig.1. Tibe inner surface DLC films deposition apparatus by Fig.2. Pictures of coplanar barrier discharge: Neon and Argon

\section{Results and discussion}




\section{Water contact angle.}

Figure 3 shows the change of the water contact angle of PE sample versus air-DBD treatment time. The water contact angle of the PE film reduces with the treatment time being extended. The water contact angle of the untreated sample is $92^{\circ}$. It dramatically decreases to $69^{\circ}$ after treatment time for one second and it continues to decrease with the treatment time increasing, which suggests the changes in the surface of PE samples mainly happen within the first second. However, the contact angle changes a little when the sample is treated for twenty seconds or more. Nai-Yi Cui et al. [12] has observed that effective plasma-induced chemical etching appears to equilibrate after $25 \%$ of the PP surface carbon is oxidized.

Contact angle measurements on treated surfaces of PE samples show the hydrophilic transformation of the polymer surfaces. The increasing of the polar molecules on the surface of the material will cause the decreasing of the contact angle, which indicates that the wettability increases. The water contact angle on the treated PE samples decreasing with the treatment time being extended suggests the hydrophilic increasing on the treated surfaces of PE samples with the treatment time being extended.
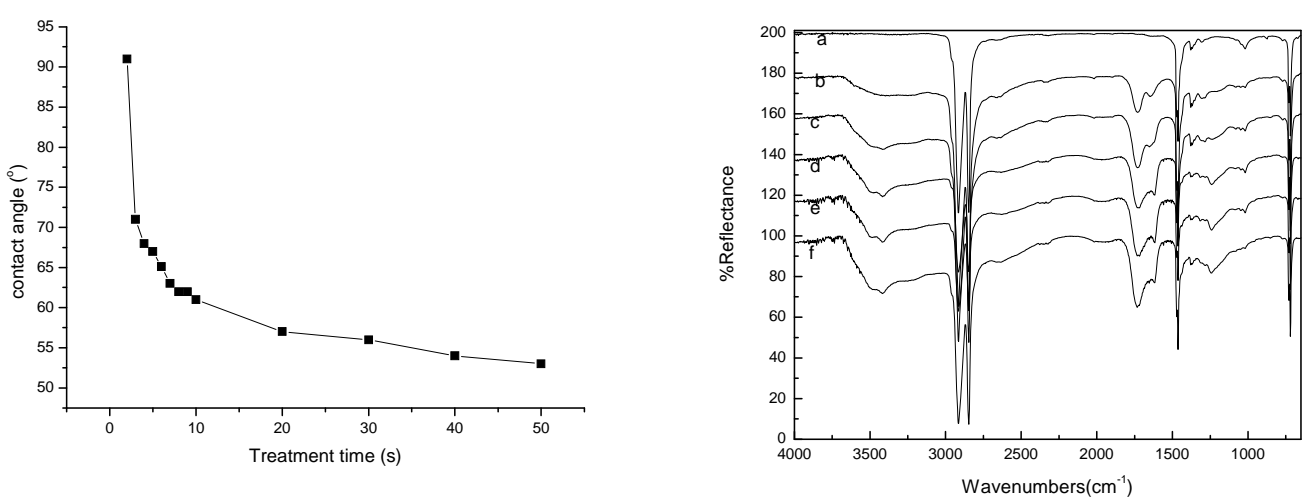

Figure 3. The variation curve of water contact angle versus the treatment time Figure 4. Tibe inner surface DLC films deposition apparatus byFTIR-ATR spectra for (a) an untreated PE film and (b) a 1s treated PE film and (c) a 10s treated PE film and (d) a 20s treated PE film and (e) a 30s treated PE film and (f) a 40s treated PE film

\section{FTIR-ATR.}

Figure 4 shows FTIR-ATR spectra for pre- and post-treated PE films. The 1s PE sample treated with the air CBD shows two new peaks at 1733 and $1646 \mathrm{~cm}-1$, while the 20s treated PE sample compared with the 1s PE sample treated with the air DBD shows two new peaks at $1242 \mathrm{~cm}-1$ and $3419 \mathrm{~cm}-1$ again. The peak at $1733 \mathrm{~cm}-1$ corresponds to $(\mathrm{C}=\mathrm{O})$ stretching vibration. The peak at 1646 cm-1 corresponds to COO - asymmetrical stretching. The peak at $1242 \mathrm{~cm}-1$ could be COOsymmetrical stretching and the peak at $3419 \mathrm{~cm}-1$ could be $-\mathrm{OH}$ group. Lehocky et al. [13] has also found similar groups on the surface of the treated PE with oxygen plasmas. Therefore, the oxidized species are introduced into the surface of PE exposed to the air CBD, which is responsible for the decrease of the contact angle on the treated sample surface. An oxygen plasma can react with the polymer surface to produce a variety of atomic oxygen functional groups, such as $\mathrm{C}-\mathrm{O}, \mathrm{C}=\mathrm{O}, \mathrm{O}-\mathrm{C}=\mathrm{O}$ at the surface [14]. Generally speaking, when polymers are exposed to plasma, two processes simultaneously happen, one is etching of the polymer surface through the reaction of atomic oxygen with the surface carbon atom, giving volatile reaction products, the other is the formation of oxygen functional groups at the polymer surface through the interaction between the active species from the plasma and the surface atom [15]. After 20s treated time, the ATR spectra of the 30s and 40s treated samples show no other peaks, and amplitude of the spectra of the 30s and 40s treated samples hardly change, which suggests the etching and oxidation come to equilibrate when the surface of samples are exposed to the air-CBD. This phenomena accords to the tendency of water contact angle with the exposed time.

XPS. 
Figure 4 shows carbon (1s) XPS spectra for (a) an untreated PE film and (b) a 20s treated PE film. Figure 4a shows the XPS spectrum of the untreated sample can be fitted with two peaks. One is the peak at binding energy $\mathrm{Eb}=285.0 \mathrm{eV}$, the other is the peak at binding energy $\mathrm{Eb}=286.3 \mathrm{eV}$. The former is the hydrocarbon signal peak, the latter should be the peak of the certain intrinsic low-level oxidized carbon group [13]. O atomic concentration is measured at $3.80 \%, \mathrm{C}$ atomic concentration is measured at $18.62 \%$ among the second peak. Figure $4 \mathrm{~b}$ shows the XPS spectrum of a 20 s treated sample. Additionally oxidized species appear and $\mathrm{O}$ atomic concentration increases. The two new peaks emerge on the high-energy side: $\mathrm{Eb}=288.0 \mathrm{eV} ; \mathrm{Eb}=289.2 \mathrm{eV}$. The former represents ketone $[-(\mathrm{C}=\mathrm{O})-]$ and/or acetal [-(O-C-O)-] carbons, the latter represents carboxyl [-(C-O)-O-] carbon.

Figure 5a shows the XPS spectrum of the untreated sample can be fitted with one peak and its binding energy is $532.8 \mathrm{eV}$. Figure $5 \mathrm{~b}$ shows the XPS spectrum of a $20 \mathrm{~s}$ treated sample. One new peak emerges and its binding energy is $534.4 \mathrm{eV}$. $\mathrm{Eb}=532.8 \mathrm{eV}$ corresponds [ $(\mathrm{C}-\mathrm{O})-]$ and $\mathrm{Eb}=534.4 \mathrm{eV}$ corresponds $[-(\mathrm{C}=\mathrm{O})-]$. The PE film only consists of carbon and hydrogen elements. The surface of $\mathrm{PE}$ film could be oxidized when it is exposed to the oxygenous environment. According to Figure 5a, $\mathrm{C}$ and $\mathrm{O}$ combine and make [-(C-O)-]. Figure.5b suggests that the active particles in plasma could break carbon-hydrogen bonds to form unsaturated carbon bonds, which combine with the ionized oxygen in discharge gas, therefore, $[-(\mathrm{C}-\mathrm{O})-]$ and $[-(\mathrm{C}=\mathrm{O})-]$ are formed in the surface of the treated PE film.
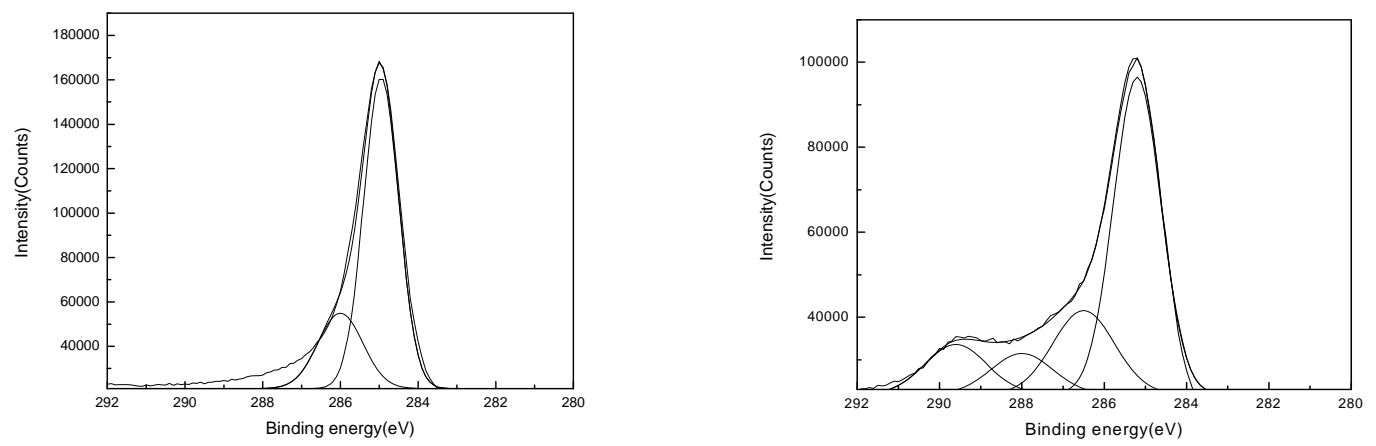

Figure 5. Carbon (1s) XPS spectra for (a) an untreated PE film and (b) a 20s treated PE film

\section{SEM.}

Figure 6 shows the morphology of the PE films with the treated time increasing. The image size is $1 \mu \mathrm{m}$. The discharge conditions are: the discharge voltage is $10 \mathrm{kV}$, the discharge frequency is $4 \mathrm{kHz}$ and the electrode gap is $2 \mathrm{~mm}$. Figure 6 (a) shows the image of the untreated PE film, which surface is smooth. Figure 6 (b) indicates the micrograph of the treated PE film for $5 \mathrm{~s}$, which surface grows rough, a lot of speculate protuberances emerge. Figure 6 (c) shows the SEM image of the treated PE film for 60s, which surface grows rougher than Figure 6 (b), much more speculate protuberances emerge. The surface the treated PE film grows rougher with the treated time increasing mainly because of the chemical etching. The chemical etching of the PE surface is through the reaction of atomic oxygen with the surface carbon atom, giving volatile reaction products, therefore a new surface layer appears.
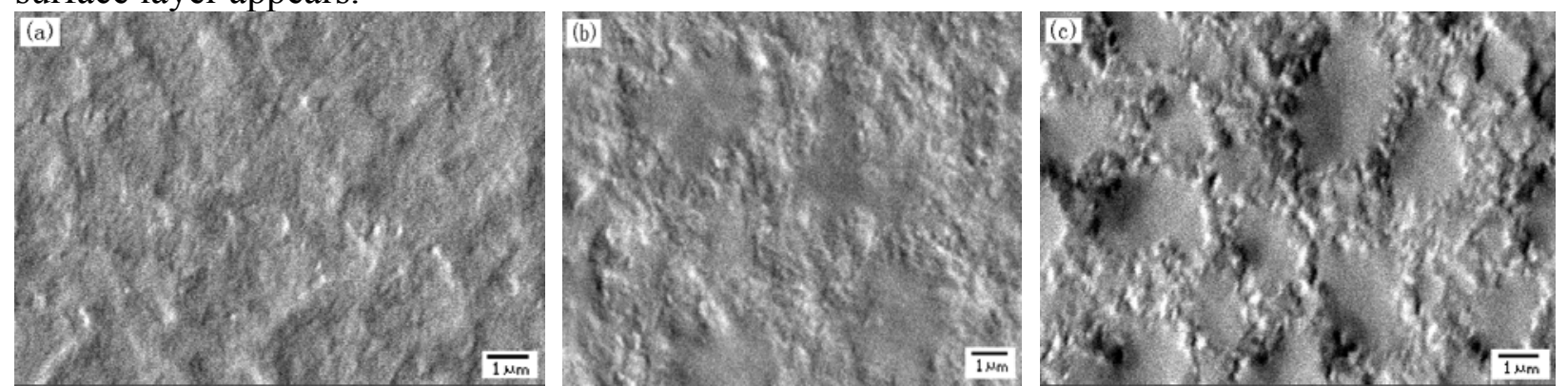

Figure 6. SEM for (a) $\mathrm{Cu}$ film deposited on an untreated PE film and (b) $\mathrm{Cu}$ film deposited on a $2 \mathrm{~m}$ treated $\mathrm{PE}$ film and (c) $\mathrm{Cu}$ film deposited on a $2 \mathrm{~m}$ treated PE film with blowing 


\section{Conclusion}

In this paper, modification of the surface properties of PE films is studied using air dielectric barrier discharge plasma at atmosphere pressure, which shows air plasma can dramatically improve wettability of PE surfaces. The treated samples are examined by water contact angle measurements, FTIR-ATR, XPS and SEM, which shows that wettability of surfaces is improved because the polar functional groups are introduced into the surface of PE samples.

\section{References}

[1] M. Cernak, J. Rahel, M. Stefecka, M. Kando. Treatment of non-woven and woven textiles using atmospheric-pressure surface discharges, Proceedings of the 17 th symposium on plasma processing, Nagasaki, Japan, pp.535-538, 2000

[2] R. Thyen, K. Hopfner, N. Klake, C.P. Klages, Cleaning of silicon and steel surfaces using dielectric barrier discharges, Plasma Polym., vol. 5, pp.91-102, 2000

[3] F. Massines, N. Gherardi, F. Sommer, Silane-based coating on polypropylene deposited by atmospheric pressure glow discharge plasmas, plasmas Polym. Vol. 5pp.151-172, 2000

[4] S. Guruvenket, G, Mohan Rao, Manoj Komath, et al, Plasma surface modification of polystyrene and polyethylene. [J] Appl. Surf. Scie. 236 (2004) 278-284

[5] S. Bhowmik, P, Jana, T.K. Chaki, et al, Surface modification of PP under different electrodes of DC glow discharge and its physicochemical characteristics. Surf. \& Coat. Tech. 185 pp.81-91, 2004

[6] R.J. Carman, R.P. Mildren. J. Phys. D: Appl. Phys. 33 (2000) L99-L103.

[7] Chen J R, Wang X Y, Wakida T. J. Appl. Polym Sci, 1998， 72: 1327 1333

[8] R. Seeb ${ }^{O} \mathrm{ck}, \mathrm{H}$. Esrom, M. Charbonnier, et al, Surface modification of polyimide using dielectric barrier discharge treatment, Surf. And Coat. Tech. 142-144 (2001) 455-459

[9] G. Borcia, C. A. Anderson, N. M. D. Brown, The surface oxidation of selected polymers using an atmospheric pressure air dielectric barrier discharge . Part I, Appl. Surf. Scie. 221 (2004) 203-214 [10] S. Ishikawa, K. Yukimura, K. Matsunaga, et al, Surf. Coat. Technol. 130 (2002) 52-56

[11] S. Meiners, J.G.H. Salge, E. Prinz, et al, Surface modification of polymer materials by transient gas discharges at atmospheric pressure. Surf. And Coat. Tech. 98 (1998) 1121-1127.

[12] Nai-Yi Cui, Norman M.D. Brown. Modification of the surface properties of a polypropylene (PP) film using an air dielectric barrier discharge plasma. Appl. Surf. Scie, 2002, 189: 31-38

[13] M. Lehocky, H. Drnovski, B. Lapcikova, et al, Colloids Surf. A 22 (2003) 125

[14] S. Guruvenket, G. Mohan Rao, Manoj Komath, et al, Plasma surface modification of polystyrene and polyethylene. [J] Appl. Surf. Scie. 236 (2004) 278-284

[15] M. Morra, E. Occhiello, F. Garbassi, Surf. Interf. Anal. 16 (1990) 412

[16] Motoki Kadowaki, Aguru Yamamoto, Shinsuke Mori, Modeling of plasma CVD on the inner surface of a microchannel, Thin Solid Films, 515, pp.4197-4202, 2007

[17] Y.H. Liu, J. Li, D.P. Liu, Properties and deposition process of a-C: H films from CH4/Ar dielectric barrier discharge plasmas, Surface \& Coating Technology, 200 pp.5819-5822, 2006

[18]D. Korzec, E. G. Finantu-dinu, Coparison of coplanar and surface barrier discharges operated in oxygen-nitrogen gas mixtures, Surface Coating Technology, vol. 174-175, pp.503-508, 2003

[19]Marcel Simor, Jozef Rahel, atmospheric-pressure diffuse coplanar surface discharge for surface treatments, Appl. Phys. Lett., vol. 81, no. 15, pp. 2716-2718, 2002 